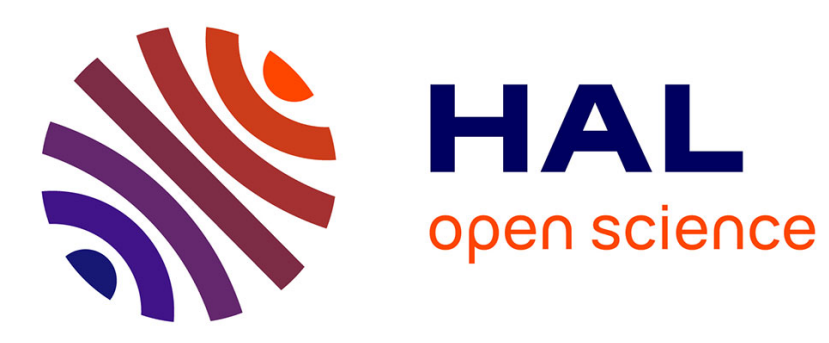

\title{
Direct perturbations of aggregate excess demand
}

Sofia B. S. D. Castro, Sami Dakhlia, Peter B. Gothen

\section{To cite this version:}

Sofia B. S. D. Castro, Sami Dakhlia, Peter B. Gothen. Direct perturbations of aggregate excess demand. 2008. halshs-00306408

\section{HAL Id: halshs-00306408 \\ https://shs.hal.science/halshs-00306408}

Submitted on 25 Jul 2008

HAL is a multi-disciplinary open access archive for the deposit and dissemination of scientific research documents, whether they are published or not. The documents may come from teaching and research institutions in France or abroad, or from public or private research centers.
L'archive ouverte pluridisciplinaire HAL, est destinée au dépôt et à la diffusion de documents scientifiques de niveau recherche, publiés ou non, émanant des établissements d'enseignement et de recherche français ou étrangers, des laboratoires publics ou privés. 


\section{Documents de Travail du Centre d'Economie de la Sorbonne}

C

E

$\mathrm{S}$

W

0

1

k

i

n

g

$\mathrm{P}$

a

$\mathrm{p}$

e

$r$

$\mathrm{S}$

CENTRE NATIONAL

DE LA RECHERCHE

SCIENTIFIQUE

Maison des Sciences Économiques, 106-112 boulevard de L'Hôpital, 75647 Paris Cedex 13

http://ces.univ-paris1.fr/cesdp/CES-docs.htm

ISSN : 1955-611X
Sofia B. S. D. CASTRO, Sami DAKHLIA, Peter B. GothEN

2008.45 


\title{
Direct perturbations of aggregate excess demand
}

\author{
Sofia B. S. D. Castro \\ Sami Dakhlia* \\ Peter B. Gothen \\ sdcastro@fep.up.pt \\ sami.dakhlia@gmail.com \\ pbgothen@fc.up.pt
}

$\diamond$ CMUP and Faculdade de Economia do Porto, Universidade do Porto, Rua Dr. Roberto Frias, 4200-464 Porto, Portugal.

* corresponding author: Department of Economics and Finance, College of Business, University of Southern Mississippi, Hattiesburg, MS 39406, USA.

- CMUP and Faculdade de Ciências, Universidade do Porto, Rua do Campo Alegre 687, 4169-007 Porto, Portugal.

\begin{abstract}
Résumé
Nous démontrons qu'une économie d'échange (définie par ses préférences et ses dotations) qui génère une fonction de demande excédentaire aggrégée (DEA) $z$ est proche de l'économie associée à la DEA $z^{\prime}$, perturbation arbitraire de $z$.
\end{abstract}

\begin{abstract}
We establish that an exchange economy, i.e., preferences and endowments, that generates a given aggregate excess demand (AED) function is close to the economy generating the AED obtained by an arbitrary perturbation of the original one.
\end{abstract}

Keywords: Arrow-Debreu, transversality, exchange economy

JEL classification: D50, C62, C02

Acknowledgements: The authors wish to thank Jean-Marc Bonnisseau, Carlos Hervès-Beloso, and John Nachbar for their very helpful comments, and in particular, Marty Golubitsky for introducing the first two authors to each other.

Part of this work was done while the first two authors were visiting CERMSEM at Paris 1 whose hospitality is gratefully acknowledged. In particular, we wish to thank Cuong Le Van for the kind invitation and Bernard Cornet for providing us with most spacious working conditions. 
Sofia Castro and Peter Gothen acknowledge support from Centro de Matemática da Universidade do Porto (CMUP) and Fundação para a Ciência e Tecnologia, through the programmes POCTI and POSI.

\section{Introduction}

Genericity results - such as establishing that the set of equilibrium prices constitutes a manifold of a certain dimension or that the number of regular equilibria is finite and stable - are obtained by perturbation techniques, where the underlying primitives (e.g., preferences and endowments) are subjected to infinitesimal changes.

Debreu's (1970) proof of local isolation of regular economies, for example, requires a perturbation of endowments. Extending the proof to critical economies requires additional effort: since the perturbation must also affect the curvature of the equilibrium manifold, a linear perturbation such as the one at the heart of Debreu's proof no longer suffices. An example of a higher-order perturbation is found in Mas-Colell (1985, Proposition 8.8.3), who shows that for a one-dimensional parameterization of economies, a "flat" AED is not generic in the first consumer's utility. It is instructive to note that Mas-Colell must resort not only to a perturbation of endowments, but also to a quadratic perturbation of (indirect) utility.

Allen (1984), on the other hand, establishes much more, namely, finiteness for multi-dimensional parameterizations of economies by employing a theorem by Tougeron (1972), according to which local finiteness of the number of pre-images is a generic property of smooth functions. The result comes at a cost, however, in that Allen works directly with AED functions rather than with the agents' underlying preferences and endowments. In this note, we show that Allen's approach is fully justified.

Specifically, we establish that the economy, i.e., preferences and endowments, that generates a given AED function is close to the economy generating the AED obtained by an arbitrary perturbation the initial one. ${ }^{1}$

We show that the Sonnenschein-Mantel-Debreu results (roughly, if it "looks" like an AED, it is an AED for some economy) are stable in the

\footnotetext{
${ }^{1}$ Lehmann-Waffenschmidt (1995) obtains a result in the same spirit for the family of one-parameterized exchange economies with an equal number of goods and consumers.
} 
following sense: the economy underlying a perturbed AED function is necessarily close to the economy behind the original AED function. Moreover, the two economies differ (slightly) in the preferences and endowments of the first consumer only.

Part of the challenge, of course, is to show that perturbations of AED functions correspond to "legal" perturbations of preferences, i.e, perturbed utility functions must continue to satisfy the canonical properties of utility functions.

This opens wider the door to further genericity and determinacy research based on transversality arguments since one may now perturb the AED function directly thus sparing one the more tedious exercise of perturbing economic primitives.

In a companion paper, Castro and Dakhlia (2008) make full use of this result to establish Thom-Boardman stratification of AED, which requires high-order perturbations that would have been more difficult to obtain by conventional means. The stratification result, in turn, provides an alternative proof of finiteness of all equilibria, including critical ones.

\section{Notation}

Consider an economy with $L$ commodities $(\ell=1, \ldots, L)$ and $I$ traders $(i=$ $1, \ldots, I)$. Let $\Omega$ be the non-negative orthant of $\mathbb{R}^{L}$ and let each trader $i$ be defined by her endowment $\omega^{i} \in \mathbb{R}^{L}$ and her preferences $\succ_{i}$, a partial order on $\Omega$ with the following properties:

1. rationality (completeness, reflexivity, and transitivity)

2. continuity $\left(\left\{x: y \succ_{i} x\right\}\right.$ and $\left\{y: y \succ_{i} x\right\}$ are open);

3. non-satiation $\left(x \geq y\left(x_{\ell} \geq y_{\ell}, \forall \ell=1, \ldots, L\right)\right.$ and $\left.x \neq y \Rightarrow x \succ_{i} y\right)$;

4. strict convexity $\left(x \sim_{i} y\right.$ and $\left.x \neq y \Rightarrow \forall \alpha \in(0,1), \alpha x+(1-\alpha) y \succ_{i} x\right)$.

Let $\Xi$ denote the space of all such preferences. Following Kannai (1970), every $\succ_{i} \in \Xi$ may be represented by a continuous utility function $u_{i}: \Omega \rightarrow \mathbb{R}$ defined as follows: for any $x \in \Omega$, there exists a unique $\hat{x}$ in the principal diagonal of $\Omega$ such that $x \sim_{i} \hat{x}$ (i.e., agent $i$ is indifferent between $x$ and $\hat{x}$ ). Then, let $u_{i}(x) \equiv\|\hat{x}\|$, where $\|$.$\| is the Euclidean norm. Denote C^{*}$ the class of utility functions thus defined. 
Let $u_{1}, u_{2} \in C^{*}$ represent the preferences $\succ_{1}, \succ_{2} \in \Xi$ of agents 1 and 2 . The metric

$$
\rho\left(\succ_{1}, \succ_{2}\right)=\max _{x \in \Omega} \frac{\left|u_{1}(x)-u_{2}(x)\right|}{1+\|x\|^{2}}
$$

induces a minimal topology on $\Xi$ with $A \equiv\{(x, y, \succ): x \succ y\}$ open in $\Omega \times \Omega \times \Xi$. Intuitively speaking, preference order $\succ_{1}$ is close to preference order $\succ_{2}$ if $\rho\left(\succ_{1}, \succ_{2}\right)$ is small. Similarly, $u_{1}$ is close to $u_{2}$ if the Euclidean distance $\left|u_{1}(x)-u_{2}(x)\right|$ is bounded by a small number for all $x$ in any compact set. $^{2}$ Finally, we define a perturbation of preferences as preferences that are distinct but arbitrarily close to the original ones. Similarly, a perturbation of a utility function is defined as a distinct, but arbitrarily close, utility function.

\section{Result}

Any preference ordering can be represented by exactly one utility function in $C^{*}$. Thus, perturbations of a $C^{*}$-utility necessarily imply perturbations of the underlying preferences and vice-versa. Moreover, direct perturbations of AED correspond to perturbations of preferences and endowments.

The following lemmas show that a perturbation of preferences corresponds to a perturbation of utility, and vice-versa.

Lemma 3.1. Consider preferences $\succ_{0} \in \Xi$ represented by a utility function $u_{0} \in C^{*}$. Let $u \in C^{*}$, representing preferences $\succ$, be a perturbation of $u_{0}$. Then $\succ \in \Xi$ is a perturbation of $\succ_{0}{ }^{3}$

Proof. Because $u$ is close to $u_{0}$, we know that for any compact subset $K \subset \Omega$,

$$
\exists \varepsilon>0:\left|u(x)-u_{0}(x)\right|<\varepsilon, \quad \forall x \in K \text {. }
$$

We have

$$
\frac{\left|u(x)-u_{0}(x)\right|}{1+\|x\|^{2}} \leq\left|u(x)-u_{0}(x)\right|<\varepsilon, \quad \forall x \in K .
$$

For $u \in C^{*}$ there exists a constant $C$, depending only on $L=\operatorname{dim} \Omega$, such that $0 \leq u(x)<C\|x\|$. (See Kannai and, in particular, his proof of Theorem

\footnotetext{
${ }^{2}$ This is just the uniform norm on compact sets, i.e., the compact-open topology.

${ }^{3}$ In fact, $\succ$ is as close to $\succ_{0}$ as $u$ is close to $u_{0}$.
} 
3.2.) We have

$$
\forall x \in \Omega, \frac{\left|u(x)-u_{0}(x)\right|}{1+\|x\|^{2}} \leq \frac{|u(x)|+\left|u_{0}(x)\right|}{1+\|x\|^{2}} \leq \frac{2 C\|x\|}{1+\|x\|^{2}},
$$

which converges to zero as $\|x\|$ approaches infinity, that is,

$$
\forall \varepsilon>0, \quad \exists R>0: \quad\|x\|>R \Rightarrow \frac{2 C\|x\|}{1+\|x\|^{2}}<\varepsilon .
$$

Take $K$ to be the compact set $C l\left(B_{R}(0)\right)$, the closure of the ball of radius $R$ about the origin.

Therefore by definition,

$$
\rho\left(\succ, \succ_{0}\right)=\max _{x \in K} \frac{\left|u(x)-u_{0}(x)\right|}{1+\|x\|^{2}}<\varepsilon .
$$

Lemma 3.2. Consider preferences $\succ_{0} \in \Xi$ represented by a utility function $u_{0} \in C^{*}$. Let $\succ \in \Xi$, represented by a utility $u \in C^{*}$, be a perturbation of $\succ_{0}$. Then $u$ is a perturbation of $u_{0}$.

Proof. We need to establish that for any compact subset $K \subset \Omega$,

$$
\forall \varepsilon>0, \quad \exists \delta>0: \quad \rho\left(\succ, \succ_{0}\right)<\delta \Rightarrow \max _{x \in K}\left|u(x)-u_{0}(x)\right|<\varepsilon .
$$

From Kannai's equation 3.2 (p. 799), we have

$$
\left\{\succ: \rho\left(\succ, \succ_{0}\right)<\delta\right\}=\left\{\succ: \max _{x \leq R} \frac{\left|u(x)-u_{0}(x)\right|}{1+\|x\|^{2}}<\delta\right\} .
$$

Since $K$ is compact, we can choose $R$ such that $K \subset B_{R}(0)$ so that $\|x\|^{2} \leq R^{2}$. Then

$$
\left|u(x)-u_{0}(x)\right|<\delta\left(1+\|x\|^{2}\right) \leq \delta\left(1+R^{2}\right) .
$$

In order to have $\left|u(x)-u_{0}(x)\right|<\varepsilon$, it suffices to choose $\delta$ such that $\delta<$ $\varepsilon /\left(1+R^{2}\right)$.

In light of these two lemmas, we can henceforth interchangeably work with preference and $C^{*}$-utility perturbations, depending on which is more convenient. 
We now turn to the excess demand of an agent endowed with $\omega^{i} \in \mathbb{R}^{L}$. Trader $i$ solves Utility Maximization Problem (UMP)

$$
\max _{x^{i} \in \Omega} u_{i}\left(x^{i}\right) \text { such that } p \cdot x^{i} \leq p \cdot \omega^{i} .
$$

Strict convexity of preferences ensures that the solution, $x^{i}\left(p, p \cdot \omega^{i}\right)$, is unique and a continuous function of both price vector $p$ and endowment $\omega^{i}$. Nonsatiation guarantees that the budget constraint is binding and can thus be written as

$$
p \cdot x^{i}=p \cdot \omega^{i} \Leftrightarrow p \cdot\left(x^{i}-\omega^{i}\right)=0 .
$$

Geometrically, the constraint is the hyperplane through $\omega^{i}$ orthogonal to $p$, while the solution to the UMP corresponds to the point of tangency between the level curves of $u_{i}$ and the hyperplane. For smooth level curves, the point of tangency is located where $\nabla u_{i}$, the gradient of $u_{i}$, is parallel to $p$. The excess demand for trader $i$ is defined as

$$
z^{i}(p)=x^{i}\left(p, p \cdot \omega^{i}\right)-\omega^{i},
$$

and the AED for the economy is given by $z(p)=\sum_{i=1}^{n} z^{i}(p)$.

The main result of this section, Theorem 3.2, establishes that a perturbation of AED is equivalent to a perturbation of economic primitives, that is, preferences and endowments. Note that, from the definition of AED, it suffices to consider the perturbation of the excess demand of a single consumer. We will thus drop the agent-specific superscript, when no confusion is possible.

We proceed by first establishing Theorem 3.1, which states that the perturbation of an agent's utility and endowment is equivalent to a perturbation of her excess demand. This will require a sequence of lemmas. Our main result (Theorem 3.2) then follows as a consequence of considering utilities in $C^{*}$.

Theorem 3.1. Consider a consumer with endowment $\omega \in \Omega$ and preferences satisfying (1)-(4) that are represented by a $C^{2}$ (twice differentiable) utility function (not necessarily in $C^{*}$ ). Let demand $x_{0}(p)$ be the unique solution to the UMP (1). Then,

(I) A solution to the UMP defined by a perturbation $(\bar{u}, \bar{\omega})$ of $(u, \omega)$ is a perturbation $\bar{x}(p)$ of $x_{0}(p)$. 
(II) Conversely, a perturbation $\bar{x}(p)$ of $x_{0}(p)$ is the unique solution of the UMP defined by a perturbation $(\bar{u}, \bar{\omega})$ of $(u, \omega)$.

Proof. The proof of part (I) is a consequence of the continuous dependence of demand on price, endowments and utility as proved in Lemma 3.3. Since strict convexity of preferences guarantees that $x_{0}(p) \equiv x_{0}(p, p \cdot \omega)$ is a continuous function of $\omega$, any perturbation of $\omega$ will correspond to a perturbation of $x_{0}(p)$. Lemma 3.3 shows that the same applies to perturbations of utility.

To prove part (II), we show that a perturbation of the solution must originate in a perturbation of utility and/or endowments. It is here that we need the assumption of a twice-differentiable utility function. At the solution $x_{0}(p)$, the budget constraint (2) is tangent to the indifference curve containing $x_{0}$, which means that the gradient of $u$ at $x_{0}, \nabla u\left(x_{0}\right)$, is orthogonal to $p \cdot(x-\omega)=0$. As such, $\nabla u\left(x_{0}\right)$ is parallel to $p$.

Let $\bar{x}(p)$ be a perturbation of $x_{0}(p)$, say $\left\|\bar{x}-x_{0}\right\|<\delta$ for small $\left.\delta\right\rangle$ 0 . If $\bar{x}$ does not satisfy the budget constraint, we perturb $\omega$ to $\bar{\omega}$ so that $p \cdot(\bar{x}-\bar{\omega})=0$. In addition, we shall choose $\bar{\omega}$ so that the hyperplane described by $p \cdot(x-\bar{\omega})=0$ is parallel to the original one. Denote the solution to the UMP defined by $u$ and the new restriction by $x_{0}^{\prime}(p)$. We have

$$
d\left(x_{0}^{\prime}, \bar{x}\right) \leq d\left(x_{0}^{\prime}, x_{0}\right)+d\left(x_{0}, \bar{x}\right),
$$

where $d\left(x_{0}, \bar{x}\right)<\delta$ and $d\left(x_{0}^{\prime}, x_{0}\right)$ can be made small by continuity of the demand with $\omega$. Since $d(\bar{\omega}, \omega) \leq d\left(x_{0}, \bar{x}\right)<\delta$, we have $d\left(x_{0}^{\prime}, x_{0}\right)<\varepsilon$ for a convenient choice of $\delta$. Hence, $\bar{x}$ is also a perturbation of $x_{0}^{\prime}$ and we can henceforth suppose that the demand for the unperturbed problem and $\bar{x}$ belong to the same budget constraint, dropping the use of the prime.

It remains to show that we can perturb the utility so that $\bar{x}(p)$ is the solution to the UMP.

Define $\Phi: \Omega \rightarrow \Omega$ by

$$
\Phi(x)=x-\varphi\left(\left\|x-x_{0}\right\|\right)\left(\bar{x}-x_{0}\right),
$$

where $\varphi: \mathbb{R} \rightarrow \mathbb{R}$ is constant and equal to 1 for $0 \leq t \leq \delta_{1}, \varphi(t)$ is constant and equal to zero for $t \geq 2 \delta_{1}$ and $\varphi(t)$ is smooth and decreasing for $\delta_{1} \leq t \leq 2 \delta_{1}$. (See Figure 1.)

We can choose $\varphi$ such that

$$
\left|\varphi^{\prime}(t)\right| \leq \frac{A}{\delta_{1}} \text { and }\left|\varphi^{\prime \prime}(t)\right| \leq \frac{B}{\delta_{1}^{2}}
$$




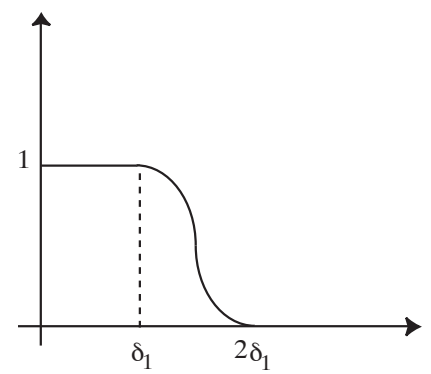

Figure 1: Graph of a function satisfying the conditions imposed on $\varphi$.

for some constants $A$ and $B$. We choose $\delta_{1}$ so that $\delta<\delta_{1}$ in order to guarantee that $\varphi\left(\left\|\bar{x}-x_{0}\right\|\right)=1$. Clearly, $\Phi$ is the identity for $\left\|x-x_{0}\right\| \geq 2 \delta_{1}$ and a translation for $\left\|x-x_{0}\right\| \leq \delta_{1}$.

Define $\bar{u}(x)=u(\Phi(x))$. We have

$$
\begin{aligned}
\bar{u}(\bar{x})=u(\Phi(\bar{x})) & =u\left(\bar{x}-\varphi\left(\left\|\bar{x}-x_{0}\right\|\right)\left(\bar{x}-x_{0}\right)\right) \\
& =u\left(x_{0}\right)
\end{aligned}
$$

and $\nabla \bar{u}(\bar{x})=\nabla u\left(x_{0}\right)$ (see Lemma 3.4 below). The level hypersurface of $\bar{u}$ at $\bar{x}$ is thus tangent to $p \cdot(x-\omega)=0$ and hence, $\bar{x}$ is a solution to the UMP defined by $\bar{u}$.

Lemmas 3.5 and 3.6 below show that $\bar{u}$ is increasing in $x$ and that its indifference curves are convex, thus ensuring that the underlying preferences satisfy (1)-(4), thereby completing the proof.

Lemma 3.3. Let $x$ be the unique solution to the UMP defined by a utility $u$ and endowment $\omega$. Then $x$ is a continuous function of $p, \omega$ and $u$.

Proof. It is clear that $x$ is a continuous function of $p$ and $\omega$. To prove continuity on $u$, we proceed by contradiction. Let $u_{n}$ be a sequence of utility functions such that as $n \rightarrow+\infty$, we have $u_{n} \rightarrow u$. Let $x_{n}=x_{n}(p)$ be the unique maximum of $u_{n}$ on

$$
H(p, \omega)=\{x: p \cdot(x-\omega)=0\} .
$$

Assume $\lim _{n \rightarrow+\infty} x_{n} \neq x$. By compactness of $H(p, \omega)$, we may assume that $x_{n} \rightarrow x^{*} \neq x_{n}$, passing to a subsequence if necessary.

Define $\delta=u(x)-u\left(x^{*}\right)$. 
Continuity of each utility in conjunction with the hypothesis that $x_{n} \rightarrow x^{*}$ implies that, for each fixed $m \in \mathbb{N}$, we have $u_{m}\left(x_{n}\right) \rightarrow u_{m}\left(x^{*}\right)$, that is, for all $\delta>0$

$$
\exists N_{1} \in \mathbb{N}: \quad n \geq N_{1} \Rightarrow\left|u_{m}\left(x_{n}\right)-u_{m}\left(x^{*}\right)\right|<\frac{\delta}{3} .
$$

In particular,

$$
u_{m}\left(x_{n}\right)-u_{m}\left(x^{*}\right)<\frac{\delta}{3} \text { i.e., } u_{m}\left(x^{*}\right)>u_{m}\left(x_{n}\right)-\frac{\delta}{3} .
$$

Let $d\left(u, u_{n}\right)<\delta / 3$, that is, for all $y \in K \subset \Omega$, a compact set also containing $x$ and $x^{*}$,

$$
\exists N_{2} \in \mathbb{N}: \quad n \geq N_{2} \Rightarrow\left|u(y)-u_{n}(y)\right|<\frac{\delta}{3} .
$$

Take $M>\max \left\{N_{1}, N_{2}\right\}$. Then, for all $y$

$$
u(y)-\frac{\delta}{3}<u_{M}(y)<u(y)+\frac{\delta}{3}
$$

In particular, replacing $y$ as appropriate, we have

$$
u_{M}\left(x^{*}\right)<u\left(x^{*}\right)+\frac{\delta}{3} \text { and }-u_{M}(x)<-u(x)+\frac{\delta}{3},
$$

which implies

$$
u_{M}\left(x^{*}\right)-u_{M}(x)<u\left(x^{*}\right)-u(x)+2 \frac{\delta}{3},
$$

i.e.,

$$
u_{M}(x)>u_{M}\left(x^{*}\right)+\frac{\delta}{3} .
$$

Using (4), we obtain

$$
u_{M}(x)>u_{M}\left(x^{*}\right)+\frac{\delta}{3}>u_{M}\left(x_{n}\right)-\frac{\delta}{3}+\frac{\delta}{3}=u_{M}\left(x_{n}\right) .
$$

Since $n \geq M$, in particular for $n=M$, we have

$$
u_{M}(x)>u_{M}\left(x_{M}\right),
$$

which is a contradiction because $x_{M}$ is the unique maximum of $u_{M}$. Hence $x^{*}=x$, proving that demand is a continuous function of utility. 
Lemma 3.4. Let $u$ and $\bar{u}$ be as defined in the proof of Theorem 3.1. Then we have $\nabla u\left(x_{0}\right)=\nabla \bar{u}(\bar{x})$.

Proof. We use the definition of $\bar{u}$ to calculate partial derivatives and obtain

$$
\frac{\partial \bar{u}}{\partial x_{j}}(x)=\sum_{i=1}^{m} \frac{\partial u}{\partial x_{i}}(\Phi(x)) \frac{\partial \Phi_{i}}{\partial x_{j}}(x)
$$

and

$$
\frac{\partial \Phi_{i}}{\partial x_{j}}(x)=\delta_{i j}-\varphi^{\prime}\left(\left\|x-x_{0}\right\|\right)\left(\bar{x}-x_{0}\right) \frac{\partial\left\|x-x_{0}\right\|}{\partial x_{j}},
$$

where $\delta_{i j}=1$ if $i=j$ and $\delta_{i j}=0$ otherwise. At $x=\bar{x}, \varphi^{\prime}\left(\left\|\bar{x}-x_{0}\right\|\right)=0$ because $\varphi\left(\left\|\bar{x}-x_{0}\right\|\right)=1$ for $0 \leq\left\|\bar{x}-x_{0}\right\| \leq \delta_{1}$. Hence,

$$
\frac{\partial \bar{u}}{\partial x_{j}}(\bar{x})=\frac{\partial \bar{u}}{\partial x_{j}}(\Phi(\bar{x}))=\frac{\partial \bar{u}}{\partial x_{j}}\left(x_{0}\right) .
$$

Lemma 3.5. Let $u$ and $\bar{u}$ be as defined in the proof of Theorem 3.1. Then $\bar{u}$ is increasing in each of its arguments.

Proof. From Lemma 3.4, we know that

$$
\frac{\partial \bar{u}}{\partial x_{j}}(x)=\sum_{i=1}^{m} \frac{\partial u}{\partial x_{i}}(\Phi(x)) \frac{\partial \Phi_{i}}{\partial x_{j}}(x)
$$

and

$$
\begin{aligned}
\frac{\partial \Phi_{i}}{\partial x_{j}}(x) & =\delta_{i j}-\varphi^{\prime}\left(\left\|x-x_{0}\right\|\right)\left(\bar{x}-x_{0}\right) \frac{\partial\left\|x-x_{0}\right\|}{\partial x_{j}} \\
& =\delta_{i j}-\varphi^{\prime}\left(\left\|x-x_{0}\right\|\right)\left(\bar{x}-x_{0}\right) \frac{x_{j}-x_{0 j}}{\left\|x-x_{0}\right\|}
\end{aligned}
$$

By choosing $\delta \ll \frac{\delta_{1}}{A}$ so that $\frac{A}{\delta_{1}} \delta \ll 1$, we have

$$
\begin{aligned}
\left|\varphi^{\prime}\left(\left\|x-x_{0}\right\|\right)\left(\bar{x}-x_{0}\right) \frac{x_{j}-x_{0 j}}{\left\|x-x_{0}\right\|}\right| & \leq\left|\varphi^{\prime}\left(\left\|x-x_{0}\right\|\right)\left(\bar{x}-x_{0}\right)\right| \\
& \leq \frac{A}{\delta_{1}} \delta \ll 1 .
\end{aligned}
$$


Again, if $\left\|x-x_{0}\right\| \leq \delta_{1}$ or $\left\|x-x_{0}\right\| \geq 2 \delta_{1}$, we have

$$
\frac{\partial \bar{u}}{\partial x_{j}}(x)=\frac{\partial u}{\partial x_{j}}(\Phi(x)) .
$$

Otherwise, $x$ is in a compact (in the annular region bounded by the discs of radius $\delta_{1}$ and $2 \delta_{1}$ ) and $\frac{\partial u}{\partial x_{j}}$ is bounded away from zero (as $u$ is increasing in each of its arguments). Hence, by making the absolute value of $\frac{\partial \Phi_{i}}{\partial x_{j}}$ sufficiently small for $i \neq j$ and close to one for $i=j$, we obtain

$$
\frac{\partial \bar{u}}{\partial x_{j}}(x)>0
$$

Lemma 3.6. The indifference curves of $\bar{u}$ as defined in the proof of Theorem 3.1 are convex.

Proof. From Thorpe [11], we know that the normal curvature of a level hypersurface of a function $u$ in the direction of a vector $v(\|v\|=1)$ perpendicular to $\nabla u(x)$ is given by

$$
-\frac{1}{\|\nabla u(x)\|}<v, H_{u}(x) v>
$$

where $H_{u}(x)$ is the Hessian of $u$ at $x$ and $\left\langle v, H_{u}(x) v\right\rangle$ represents the quadratic form defined by $H_{u}(x)$ (see exercise 2.1 in [11] and section 2 of Gladiali and Grossi [5]).

Because the level curves of $u$ are convex, we know that $H_{u}(x)$ is positive definite for all $x$. We show that the entries of $H_{\bar{u}}(x)$ are close to the entries of $H_{u}(\Phi(x))$. Hence, $\bar{u}$ can be chosen so that $H_{\bar{u}}(x)$ is also positive definite.

By differentiating the first derivatives obtained in the proof of Lemma 
3.4, we obtain

$$
\begin{aligned}
\frac{\partial^{2} \bar{u}(x)}{\partial x_{k} \partial x_{j}}= & \frac{\partial}{\partial x_{k}}\left[\sum_{i=1}^{m} \frac{\partial u}{\partial x_{i}}(\Phi(x)) \frac{\partial \Phi_{i}}{\partial x_{j}}(x)\right]= \\
= & \sum_{i, l} \frac{\partial^{2} u}{\partial x_{l} \partial x_{i}}(\Phi(x)) \frac{\partial \Phi_{l}(x)}{\partial x_{k}} \frac{\partial \Phi_{i}(x)}{\partial x_{j}}+\sum_{i} \frac{\partial u}{\partial x_{i}}(\Phi(x)) \frac{\partial^{2} \Phi_{i}(x)}{\partial x_{k} \partial x_{j}}= \\
= & \frac{\partial^{2} u}{\partial x_{k} \partial x_{j}}(\Phi(x))+\frac{\partial^{2} u}{\partial x_{k} \partial x_{j}}(\Phi(x))\left[\varphi^{\prime}\left(\left\|x-x_{0}\right\|\right)\right]^{2} . \\
& .\left(\bar{x}-x_{0}\right)_{j}\left(\bar{x}-x_{0}\right)_{k} \frac{\partial\left\|x-x_{0}\right\|}{\partial x_{k}} \frac{\partial\left\|x-x_{0}\right\|}{\partial x_{j}}+ \\
& +\sum_{i \neq j, l \neq k} \frac{\partial^{2} u}{\partial x_{l} \partial x_{i}}(\Phi(x)) \frac{\partial \Phi_{l}(x)}{\partial x_{k}} \frac{\partial \Phi_{i}(x)}{\partial x_{j}}+\sum_{i} \frac{\partial u}{\partial x_{i}}(\Phi(x)) \frac{\partial^{2} \Phi_{i}(x)}{\partial x_{k} \partial x_{j}}
\end{aligned}
$$

Note that

$$
\frac{\partial^{2} \Phi_{i}(x)}{\partial x_{k} \partial x_{j}}
$$

depends on terms of the form $\left(\bar{x}-x_{0}\right)_{j}$. Note also that for $x$ such that either $0 \leq\left\|x-x_{0}\right\| \leq \delta_{1}$ or $\left\|x-x_{0}\right\| \geq 2 \delta_{1}$, the derivatives of $\varphi$ are zero (because $\varphi$ is constant) and therefore, the second derivatives of $u$ and $\bar{u}$ coincide. Since

$$
\left\{x \in \Omega: \delta_{1} \leq\left\|x-x_{0}\right\| \leq 2 \delta_{1}\right\}
$$

is compact, the derivatives of $u$ are bounded. We may then use our choice of $\delta<\delta_{1}$ to bound the remaining derivatives in the same way as in the proof of Lemma 3.5, so that the products involving derivatives of $\varphi$, and hence of $\Phi$ become small.

We can now state our main result.

Theorem 3.2. Let $z_{0}(p)$ be the AED for an economy with $L$ goods and $I$ traders characterized by preferences $\succ_{0}^{i}$ satisfying (1)-(4) and endowments $\omega_{0}^{i}, i=1, \ldots, L$. An $A E D z(p)$ is a perturbation of $z_{0}(p)$ if and only if $z(p)$ is the AED for an economy with $L$ goods and I consumers such that the new preferences $\succ^{1}$ of the first consumer are perturbations of $\succ_{0}^{1}$ and the new endowments $\omega^{1}$ are perturbations of $\omega_{0}^{1}$. 
Proof. Choose utilities in $C^{*}$ to represent the preferences. Using Lemma 3.8, we can approximate these by $C^{2}$ utilities. Moreover, because of Lemmas 3.1 and 3.2, perturbations in utility correspond to perturbations in preferences and vice-versa. The result then follows from Theorem 3.1 and in a straightforward way from the definition of AED.

Note that the preferences represented by the $C^{2}$ utilities obtained through Theorem 3.1 can be described by utilities in $C^{*}$.

In the proof of Lemma 3.8, we need the following:

Lemma 3.7. Let $f: \mathbb{R}^{n} \rightarrow \mathbb{R}$ be continuous and such that $x>y \Rightarrow f(x)>$ $f(y)$, where $x>y$ means, as usual, that

$$
\exists i: \quad x_{i}>y_{i} \text { and } x_{j} \geq y_{j}, \text { for } i \neq j .
$$

Then $f$ is strictly quasi-concave if and only if $f$ is strictly concave on level sets, that is, if $x_{1}, x_{2}$ are such that $f\left(x_{1}\right)=f\left(x_{2}\right)$ we have

$$
\forall \lambda \in[0,1]: f\left(\lambda x_{1}+(1-\lambda) x_{2}\right)>\lambda f\left(x_{1}\right)+(1-\lambda) f\left(x_{2}\right) .
$$

Proof. The if part of the statement is trivial. To show the converse, suppose that (5) holds. Note that $\lambda f\left(x_{1}\right)+(1-\lambda) f\left(x_{2}\right)=f\left(x_{1}\right)=f\left(x_{2}\right)$. We want to show that

$$
\forall \lambda \in(0,1) \forall x, y \quad f(\lambda x+(1-\lambda) y)=f\left(y_{\lambda}\right)>\min \{f(x), f(y)\},
$$

which holds trivially if $f(x)=f(y)$. Let $f(x) \neq f(y)$ and assume $f(x)<$ $f(y)$. Denote by $L_{z}=\{x: f(x)=f(z)\}$ the level set of $z$. By monotonicity, $L_{x} \cap L_{y}=\emptyset$.

Take $w \in L_{y}$ such that $w>x$, that is, $f(w)>f(x)$. Consider the points on the segment connecting $x$ to $w$,

$$
w_{t}=t x+(1-t) w, \text { for } t \in[0,1] .
$$

By monotonicity, $L_{w_{t}}$ lies between $L_{x}$ and $L_{w}$ and $f\left(w_{t}\right)>f(x)$. Since $y_{\lambda} \in L_{w_{t}}$ for some $t$, we have $f\left(y_{t}\right)>f(x)$, concluding the proof.

Lemma 3.8. Let $u \in C^{*}$ be a utility representing preferences that satisfy (1)-(4). Endow $C^{*}$ with the uniform norm on compact sets. There exists $\bar{u}$ which is $C^{2}$ and close to $u$ representing preferences that satisfy (1)-(4). 
Proof. We need to show that $\bar{u}$ is strictly quasi-concave and increasing in each coordinate.

We use a convolution kernel or mollifier, $\theta_{\varepsilon}: \Omega \rightarrow \mathbb{R}$, as in Hirsch [6], chapter 2, or Ghomi [4]. The mollifier is a non-negative function which takes the value zero outside a ball of radius $\varepsilon>0$ and such that

$$
\int_{\Omega} \theta_{\varepsilon}=1
$$

An explicit construction for such a $\theta_{\varepsilon}$ is given in Ghomi [4]. Define

$$
\bar{u}(x)=\int_{\Omega} u(x-y) \theta_{\varepsilon}(y) d y .
$$

Theorem 2.3 in Hirsch [6] asserts that $\bar{u}$ and $u$ are $\varepsilon$-close on compact sets. Since $u$ is strictly quasi-concave, using Lemma 3.7, we have, for $\lambda \in[0,1]$,

$$
\begin{aligned}
\bar{u}\left(\lambda x_{1}+(1-\lambda) x_{2}\right) & =\int_{\Omega} u\left(\lambda x_{1}+(1-\lambda) x_{2}-y\right) \theta_{\varepsilon}(y) d y \\
& =\int_{\Omega} u\left(\lambda\left(x_{1}-y\right)+(1-\lambda)\left(x_{2}-y\right)\right) \theta_{\varepsilon}(y) d y \\
& >\int_{\Omega}\left[\lambda u\left(\left(x_{1}-y\right)+(1-\lambda) u\left(x_{2}-y\right)\right] \theta_{\varepsilon}(y) d y\right. \\
& =\lambda \bar{u}\left(x_{1}\right)+(1-\lambda) \bar{u}\left(x_{2}\right) .
\end{aligned}
$$

Since monotonicity is preserved by integration, $\bar{u}$ is monotonous and, by Lemma 3.7 , it is strictly quasi-concave. ${ }^{4}$

\section{References}

[1] Allen, B., 1984, "On the finiteness of the equilibrium price set." $C A-$ RESS Working Paper \#85-03, University of Pennsylvania.

[2] Castro, S.B.S.D. and S. Dakhlia, 2008, "Thom-Boardman stratification of aggregate excess demand and finiteness of equilibria." Cahiers du Centre d'Economie de la Sorbonne, Université Panthéon-Sorbonne (Paris 1).

[3] Debreu, G., 1970, "Economies with a finite set of equilibria." Econometrica $38,387-392$.

\footnotetext{
${ }^{4}$ For concave utility functions, an analogous result was obtained by Kannai (1974).
} 
[4] Ghomi, M., 2002, "The problem of optimal smoothing of convex functions." Proceedings of the American Mathematical Society 130 (8), $2255-2259$.

[5] Gladiali, F. and M. Grossi, 2004, "Strict convexity of level sets of solutions of some nonlinear elliptic equations." Proceedings of the Royal Society of Edinburgh 134A, 363-373.

[6] Hirsch, M., 1976, Differential Topology, Graduate Texts in Mathematics 33 (Springer-Verlag, New York).

[7] Kannai, Y., 1970, "Continuity properties of the core of a market." Econometrica 38 (6), 791-815.

[8] Kannai, Y., 1974, "Approximation of convex preferences." Journal of Mathematical Economics 1, 101-106.

[9] Lehmann-Waffenschmidt, M., 1995, "On the equilibrium price set of a continuous perturbation of exchange economies." Journal of Mathematical Economics 24, 497-519.

[10] Mas-Colell, A., 1985, The Theory of General Economic Equilibrium: a Differentiable Approach (Cambridge University Press).

[11] Thorpe, J.A., 1979, Elementary Topics in Differential Geometry, Ergebnisse Band 71 (Springer Verlag, New York).

[12] Tougeron, J.C., 1972, Idéaux de Fonctions Différentiables (Springer Verlag, New York). 